\title{
Monitoreando la DEMOCRACIA: Profundizando un CONSENSO EMERGENTE*
}

\author{
Gerardo L. Munck \\ Universidad del Sur de California, Estados Unidos
}

\begin{abstract}
Resumen
Este trabajo considera el monitoreo de la democracia y aborda cuatro preguntas: ¿Por qué debe monitorearse la democracia? ¿Quiénes son los monitores? ¿Qué debe monitorearse? Y, ¿cómo debe llevarse a cabo el monitoreo? Primero articula el consenso emergente referido al monitoreo de la democracia. Luego la discusión se vuelca a varios nuevos desafíos que necesitan ser resueltos. Específicamente, el artículo se focaliza en los desafíos de reconocer y balancear múltiples valores políticos, reunir a políticos con investigadores, integrar esfuerzos de monitoreo y solucionar problemas de medición. Se pone énfasis en la importancia de plantear estas temáticas complejas y de profundizar el consenso surgido en referencia al monitoreo de la democracia.
\end{abstract}

\begin{abstract}
This paper considers efforts to monitor democracy and addresses four questions: Why should democracy be monitored? Who are the monitors? What is to be monitored? And, how is monitoring to be conducted? It first highlights and articulates an emerging consensus regarding the monitoring of democracy. Subsequently the discussion turns to a set of new challenges that remain to be resolved. Specifically, the paper focuses on the challenges of balancing multiple political values, bringing politicians and researchers together, integrating monitoring efforts, and resolving measurement problems. Emphasis is placed on the importance of tackling these complex issues and hence on deepening the consensus that has emerged regarding the monitoring of democracy.
\end{abstract}

\section{PALABRAS CLAVE • Democracia • Monitoreo • Medición}

\section{INTRODUCCIÓN}

Luego del final de la Guerra Fría, la promoción de la democracia se volvió una preocupación para un amplio espectro de actores y, como parte inherente de muchos programas de promoción de la democracia, se realizaron esfuerzos concertados para monitorear la democracia en países alrededor del mundo. El monitoreo de la democracia se concentró inicialmente en la observación electoral, una actividad que logró atención internacional a partir del trabajo de las Naciones Unidas, la Organización de Estados Americanos y el Centro Carter en las elecciones de Nicaragua en 1990. Sin embargo, rápidamente el alcance de las actividades de monitoreo se expandió más allá de las elecciones y comenzó a incluir un conjunto de temáticas directa o

\footnotetext{
*Agradezco los comentarios de Staffan Lindberg y Sebastián Mazzuca.
} 
estrechamente relacionadas con la democracia, tales como las fuentes de financiamiento de los partidos políticos, el acceso público a la información y la libertad de prensa, la corrupción de funcionarios públicos, el funcionamiento del poder judicial, el respeto a los derechos humanos, y la implementación de políticas públicas. En la actualidad, una década y media luego del colapso del Muro de Berlín, la producción y diseminación de información acerca de la democracia, muchas veces concebida en forma muy amplia, se ha vuelto una cuestión rutinaria. Y, como consecuencia, ciudadanos alrededor del mundo tienen fácil acceso a evaluaciones bastante completas del estado de la democracia en sus países.

Este trabajo, parte de un programa de investigación más amplio ${ }^{1}$, intenta aportar de dos maneras a este esfuerzo colectivo de monitoreo de la democracia. En primer lugar, resalta y articula el consenso emergente referido al monitoreo de la democracia. Específicamente, el trabajo se concentra en cuatro preguntas: ¿Por qué debe monitorearse la democracia? ¿Quiénes son los monitores? ¿Qué debe monitorearse? Y, ¿cómo debe llevarse a cabo el monitoreo? e intenta mostrar que se ha logrado un importante nivel de consenso en lo referido a las mismas. En segundo lugar, argumenta que, a pesar de importantes avances, aún persisten muchas cuestiones complejas por ser abordadas en lo que se refiere a las preguntas enunciadas aquí -el por qué, quiénes, qué y cómo del monitoreo- y que es vital que tales cuestiones sean tratadas y resueltas. Efectivamente, para asegurar que se mantenga el ímpetu que se ha desarrollado en torno al monitoreo de la democracia en los últimos años, es necesario profundizar el consenso que ha surgido en referencia al mismo.

\section{II. ¿CUÁL ES LA SITUACIÓN ACTUAL? UN CONSENSO EMERGENTE}

\section{1. ¿Por qué monitorear la democracia?}

La razón para monitorear la democracia es, en primer lugar, el difundido consenso de que la democracia es un valor universal ${ }^{2}$. Esta perspectiva es explícitamente proclamada y endosada en documentos hito, desde la Declaración y el Programa de Acción de Viena, adoptada por la Conferencia Mundial sobre Derechos Humanos en 1993, y la Declaración del Milenio, puesta en marcha por la Asamblea General de las Naciones Unidas (ONU) en 2000, hasta el Tratado de Amsterdam, firmado por la Unión Europea (UE) en 1997, y la Carta Democrática Interamericana, suscrita por la Asamblea General de la Organización de Estados Americanos (OEA) en $2001^{3}$. Sin embargo, estando ausente algún mecanismo para el monitoreo de los valores incluidos en

1 Mis evaluaciones del estado del arte sobre la medición de la democracia y conceptos relacionados, junto con varias propuestas para el mejoramiento de las mediciones, incluyen Munck y Verkuilen (2002) y Munck (2005 y 2006). Mis esfuerzos por construir un nuevo Índice de Democracia Electoral para el Programa de las Naciones Unidas para el Desarrollo (PNUD) se presentan en el informe La Democracia en América Latina (PNUD, 2004: 21-30 del compendio estadístico).

2 Sobre la universalidad del valor de la democracia, ver Sen (2001). Para una visión expansiva de las virtudes de la democracia, ver Dahl (1999: Capítulo 5); para una defensa más modesta de la democracia, ver Przeworski (1997).

3 Asamblea General de las Naciones Unidas (1993: Artículos 1 y 8; y 2000: Sección V), Unión Europea (1997: Artículo 6), y Organización de los Estados Americanos (2001: Artículo 1). Sobre el incremento destacado de la democracia en el derecho internacional y la difusión de "cláusulas democráticas", ver Rich (2001) y Piccone (2005). 
estos documentos, estos corren el riesgo de ser vistos como declaraciones vacías de intención, pues, dado que el monitoreo tiene por objetivo generar mediciones, o datos, y dado que ni los gobernantes pueden formular políticas basadas en conocimientos sólidos ni los ciudadanos pueden hacer que sus gobernantes rindan cuentas sin información sistemática y confiable sobre el estado de la democracia, el monitoreo puede ayudar a promover valores ampliamente compartidos y a "inculcar sustancia en frases maravillosas"4. En efecto, al proverbio "sólo puedes administrar aquello que puedes medir", se podría incluir el addendum que sólo puedes hacer que aquéllos que administran los asuntos públicos rindan cuentas si puedes medir su desempeño.

\section{2. ¿Quiénes son los monitores?}

Más allá de declaraciones que reafirman un compromiso con el valor de la democracia, un amplio espectro de actores ha adoptado la promoción de la democracia como una causa y han otorgado fuerza a tales declaraciones al involucrarse en ejercicios de monitoreo. La promoción de la democracia se inició en gran medida como una iniciativa del gobierno de Estados Unidos y organizaciones no-gubernamentales localizadas en la misma nación durante los años de la administración Reagan. En 1983, se estableció la Fundación Nacional para la Democracia (NED) y ese mismo año se fundaron el Instituto Nacional Demócrata para Asuntos Internacionales (NDI) y el Instituto Republicano Internacional (IRI), dos institutos ligados a los partidos políticos de mayor importancia de EE.UU. y financiados por el NED. El Centro Carter, creado en 1982, y el IFES (anteriormente denominado Fundación Internacional para Sistemas Electorales), abierto en 1987, fueron otros de los actores importantes que se involucraron en la promoción de la democracia en forma temprana ${ }^{5}$.

Tras el fin de la Guerra Fría, la promoción de la democracia continuó siendo una preocupación central para el gobierno estadounidense. La Oficina de Democracia y Gobernabilidad de la Agencia de Estados Unidos para el Desarrollo Internacional (USAID) se estableció en 1994, y el Buró de la Democracia, los Derechos Humanos y el Trabajo se conformó el mismo año, producto de un proceso de reorganización, dentro del Departamento de Estado de Estados Unidos. Este Departamento continuó publicando sus Informes por Países sobre Prácticas de Derechos Humanos, una serie que comenzó en 1977, y lanzó el nuevo Informe Anual sobre Libertad Religiosa Internacional en 1999. Finalmente, la Corporación del Desafío del Milenio (MCC) se estableció dentro del mismo Departamento de Estado para administrar la Cuenta del Desafío del Milenio (MCA) en 2004. Pero con el comienzo de la década del noventa la promoción de la democracia se volvió una cuestión global.

\footnotetext{
Esta frase es una adaptación del título de un artículo de Pastor (2003).

Varios precedentes del interés por el monitoreo de la democracia que surge en los años de Reagan merecen mencionarse. Organizaciones de derechos humanos, como la londinense Amnistía Internacional, fundada en 1961, y la estadounidense Human Rights Watch, fundada en 1978, desempeñaron un rol pionero para llamar la atención sobre las violaciones de los derechos asociados con valores políticos centrales. Además, ha habido numerosas iniciativas para generar datos sobre política y democracia en las universidades de Estados Unidos desde la década de 1960, incluyendo el trabajo de Arthur Banks, quien comenzó a publicar el ampliamente utilizado y regularmente actualizado Cross-National Time-Series Data Archive en 1968, y Ted Robert Gurr, el diseñador original de la serie Polity, publicada por primera vez en 1978. Paralelamente, la organización estadounidense Freedom House, fundada en 1941, comenzó a publicar evaluaciones anuales del estado de la libertad en todo el mundo en 1972. Para una descripción de múltiples esfuerzos por generar datos sobre asuntos políticos, ver Scheuch (2003).
} 
Organizaciones intergubernamentales regionales adoptaron la promoción de la democracia decisivamente. En Europa, la Organización para la Seguridad y la Cooperación en Europa (OSCE) constituyó en 1990 la Oficina de Elecciones Libres, que más tarde se transformó en la Oficina de Instituciones Democráticas y de Derechos Humanos, y en 1997 la posición de Representante para la Libertad de los Medios de Comunicación. El Consejo de Europa estableció la Comisión Europea para la Democracia a Través de la Ley -también conocida como la Comisión de Venecia- en 1990. Y la Iniciativa Europea para la Democracia y los Derechos Humanos de la Unión Europea se creó en 1994. En las Américas, la Organización de Estados Americanos (OEA) estableció la Unidad para la Promoción de la Democracia, subsecuentemente renombrada como la Oficina para la Promoción de la Democracia, en 1990. Y la Comisión Interamericana de Derechos Humanos de la OEA, formada anteriormente en 1959, publicó con regularidad una serie de informes y creó la Oficina del Relator Especial para la Libertad de Expresión en 1997.

Asimismo, organizaciones globales intergubernamentales se volvieron actores importantes en la promoción de la democracia en la era de la post-Guerra Fría. La División de Asistencia Electoral de la ONU, denominada en un principio Unidad de Asistencia Electoral, se estableció en 1991. La Oficina del Alto Comisionado para los Derechos Humanos de la ONU y el puesto de Relator Especial para la Libertad de Opinión y Expresión de la ONU se crearon en 1993. Y el Programa de las Naciones Unidas para el Desarrollo (PNUD), junto con su Informe sobre el Desarrollo Humano, progresivamente se enfocó más y más en temas sobre la gobernabilidad democrática con el correr de la década del 90.

En suma, un rango de actores de diferentes tipos han perseguido activamente la promoción y monitoreo de la democracia ${ }^{6}$. Sin lugar a duda, existe alguna duplicación de esfuerzos, a medida que más actores se han volcado hacia una actividad que se ha puesto de moda. Pero esto no ha sido un problema: dado el actual estado de conocimiento, el beneficio de poseer múltiples perspectivas seguramente sobrepasa los costos en recursos duplicados. De hecho, el número y la diversidad de los actores comprometidos con el monitoreo de diversos aspectos de la democracia son un signo de la salud de, y el estatus de legitimidad que se otorga a, los ejercicios de monitoreo. $\mathrm{Y}$, al constituir una red extensa y activa, estos actores han dado fuerza al compromiso asumido en declaraciones acerca del valor de la democracia.

\section{3. ¿Qué debe monitorearse?}

Las prácticas cambiantes de estos actores también han contribuido a clarificar qué debe ser monitoreado, lo cual involucra la complicada cuestión de cómo definir el concepto tan usado y discutido de democracia. En primer lugar, las experiencias de observación electoral han fortalecido la comprensión de que las elecciones son un proceso que van más allá del evento central del día en que los electores hacen uso del derecho al voto. Pues, cada vez más, el monitoreo ha abordado un conjunto de temáticas que afectan el proceso electoral, tales como las barreras legales para la entrada al proceso electoral, el financiamiento partidario y de campaña, la libertad de prensa y de expresión, y la independencia de los tribunales electorales.

$6 \quad$ El listado de actores mencionado aquí no es exhaustivo. En particular, el número de ONGs involucradas en la promoción de la democracia es extremadamente amplio. Para un listado más completo y una discusión de los actores relevantes, ver Bjornlund (2004: Capítulos 2 y 4) y Campbell y Carroll (2005). 
En segundo lugar, aunque en gran medida el monitoreo comenzó con, y continua estando muy enfocado en, la observación electoral; también es ampliamente aceptado en la actualidad que la democracia involucra más que las elecciones, como se enfatiza en el informe del PNUD sobre La Democracia en América Latina (PNUD, 2004: Sección 1). En este sentido, los monitores de la democracia abordan rutinariamente una variedad de derechos políticos y civiles, y ahondan en preguntas tales como los canales para la participación ciudadana más allá de la elección de los funcionarios públicos, los "frenos y contrapesos" entre las diversas ramas de gobierno, el control civil de las Fuerzas Armadas, la corrupción de los funcionarios públicos, la administración de justicia, la protección de la vida, la igualdad de los ciudadanos ante a la ley y el derecho a la propiedad privada.

Pues los monitores han gravitado gradualmente hacia una concepción de la democracia que incluye, como una parte indispensable, un enfoque en las elecciones, pero que también va más allá de las elecciones. Y, en un grado importante, se ha desarrollado un consenso implícito y amplio en lo que se refiere a un número de elementos que debe incluirse en una evaluación exhaustiva del estado de la democracia. En efecto, aun aquéllos que defienden una definición minimalista de la democracia reconocen que una evaluación de la calidad de la democracia debe tomar en cuenta una amplia gama de derechos ciudadanos.

\section{4. ¿Cómo debe llevarse a cabo el monitoreo?}

Finalmente, en referencia a cómo debe llevarse a cabo el monitoreo, se ha progresado mucho en los últimos quince años. El trabajo de académicos, organizaciones no-gubernamentales, organizaciones intergubernamentales y agencias de desarrollo, ha ofrecido indicios importantes acerca de cómo deberían abordarse cuestiones metodológicas centrales tales como la selección de indicadores, esto es, las contrapartes observables de los conceptos que se usan para operacionalizar los conceptos, e incluso la identificación de umbrales para distinguir variados grados de falencias. Tomados en conjunto, estos esfuerzos representan aportes importantes hacia la construcción de instrumentos de medición ${ }^{7}$. Además, se han generado datos sobre un rango de diferentes aspectos de la democracia, así como también sobre cuestiones estrechamente relacionadas con ella, y el debate en torno a estos intentos ha rendido algunas valiosas lecciones en relación a cómo abordar varios problemas de medición ${ }^{8}$. Y el progresivo refinamiento de prácticas ha permitido que, por lo menos con respecto a un aspecto de la democracia -los procesos electorales- se ha dado el paso importante de especificar ciertos estándares comunes para ser usados en las actividades de monitore $0^{9}$.

9 Ver la Declaración de Principios para la Observación Internacional de Elecciones y Código de Conducta para Observadores Internacionales de Elecciones, un acuerdo promovido por el Centro Carter, la División de Asistencia Electoral de la ONU y el Instituto Nacional Demócrata para Asuntos Internacionales (NDI), y endosado por 21 organizaciones el 24 de octubre de 2005. 


\section{III. ¿CUÁLES SON LOS DESAFÍOS? PROFUNDIZANDO EL CONSENSO}

Es importante reconocer, en vista de los desarrollos discutidos antes, que aunque los intentos de monitoreo de la democracia son relativamente nuevos, ha surgido un consenso en lo referente a un número de cuestiones clave. Se ha logrado mucho en un corto periodo de tiempo y, por lo tanto, los futuros esfuerzos cuentan con mucho sobre lo cual construir. Sin embargo, también es crucial reconocer que permanecen muchos desafíos fundamentales que deben ser confrontados en lo que se refiere a las preguntas tratadas aquí -el por qué, quiénes, qué y cómo del monitoreo- y que mucho depende de la forma en que estos desafíos son abordados. Por cierto, la sustentabilidad de los esfuerzos de monitoreo probablemente dependa del desarrollo de alguna forma de acuerdo sobre cómo estas cuestiones complejas deben ser solucionadas y, por lo tanto, de la profundización del consenso que ha surgido concerniente al monitoreo de la democracia.

\section{1. ¿Por qué? Reconociendo y balanceando múltiples valores políticos}

Un primer desafío se refiere al reconocimiento de, y balance entre, múltiples valores políticos. Cómo se mencionó anteriormente, la promoción y el monitoreo de la democracia se justifica por el estatus de la misma como valor universal. Pero la democracia ciertamente no es el único valor que vale la pena defender ${ }^{10}, \mathrm{y}$, por lo tanto, un énfasis sobre la democracia debe tomar en consideración, y ser balanceado contra, otros valores. Esto, por supuesto, abre la puerta a argumentos cuyo objetivo es debilitar la causa de la democracia. De hecho, un obstáculo potencial que enfrentan los esfuerzos internacionales a favor de la democracia es el principio de no-intervención en los asuntos de países soberanos. Y el argumento que la democracia es un asunto secundario, frente a objetivos más urgentes como el desarrollo, es frecuentemente expresado en discusiones políticas, incluso por aquéllos que proclaman estar a favor de la democracia. Pero es importante confrontar estos argumentos y no desecharlos prematuramente.

Muchas de las críticas a la democracia pueden responderse de manera que clarifiquen por qué es justificable promover la democracia. Con respecto a la no-intervención, la evolución reciente de prácticas en la arena mundial muestra que la soberanía no es un principio absoluto. Esto es muy claro en casos extremos de crisis humanitaria y de Estados Fallidos (Comisión Internacional sobre Intervención y Soberanía de los Estados, 2001; Krasner, 2004). Pero también lo es en el caso de las actividades rutinarias y ordinarias como el monitoreo electoral (Pastor, 2003: 16-18; McFaul, 2004: 53-55). Efectivamente, en los últimos años hemos sido testigos de un creciente número de precedentes de intervención, tanto consensuada como coercitiva, en nombre de la democracia. A su vez, en lo que respecta al desarrollo, investigaciones académicas recientes han demostrado que el argumento que los regímenes autoritarios tienen un desempeño económico superior a la democracia y que, por lo tanto, es deseable postergar las demandas por la democracia, carece de fundamentos empíricos (Przeworski, Alvarez, Cheibub y Limongi, 2000; PNUD 2002: Capítulo 2). 
Pero algunos de los puntos expresados por críticos y escépticos tienen algo de verdad y no deben ser enteramente desestimados. No es imprudente cuestionar el recurso a la intervención forzada y requerir que aquéllos que se proponen restringir la soberanía de un país en nombre de la democracia asuman la carga de la prueba y demuestren que la intervención no es mayormente, o incluso parcialmente, una fachada para los intereses de algún otro país, y que es probable que traiga los resultados buscados. Además, no es absurdo argumentar que múltiples valores no pueden lograrse simultáneamente y, por lo tanto, que es necesario pensar con detención sobre la prioridad que se le da a distintos valores y sobre las implicancias políticas que resultan de la ponderación de los valores.

Por lo tanto, los promotores de la democracia deben hacer más para abordar preguntas sobre el valor de la democracia en relación con otros valores políticos. Al igual que la soberanía, la democracia no es un valor absoluto, y aceptar esto no implica que la democracia se devalúe. Por el contrario, el caso a favor de la democracia se fortalecerá en tanto se base en argumentos más precisos y bien fundados, y el futuro de la democracia estará mejor asegurado en tanto la democracia se promueva sin desatender a otros valores.

\section{2. ¿Quiénes? Reuniendo a políticos con investigadores}

Un segundo reto consiste en la tarea de reunir a políticos e investigadores. Como se discutió anteriormente, un conjunto de organizaciones se encuentran activas en el campo de la promoción de la democracia. Estas organizaciones están conformadas por personal diverso con antecedentes, orientaciones y aptitudes variadas, tales como oficiales gubernamentales y representantes públicos, líderes partidarios y activistas, funcionarios públicos nacionales e internacionales, asesores políticos y consultores, y activistas sociales. Por lo tanto, en la medida en que estas organizaciones se involucran en ejercicios de monitoreo, y persiguen el objetivo de mejorar tanto la calidad de la información que generan como la utilidad política de esta información, deben concebir maneras de coordinar sus actividades políticas y de investigación o, para decirlo de una manera diferente, de combinar el trabajo de su personal de perfiles político y de investigación.

Hacer que información rigurosa y creíble tenga incidencia e impacto sobre la política, la novedad clave en los esfuerzos por monitorear la democracia, demanda cosas diferentes de políticos e investigadores. Desde la perspectiva de los políticos, requiere un compromiso previo tanto de no intervención en, como de aceptación de los resultados de, los ejercicios de monitoreo. De hecho, el objetivo principal del monitoreo se pierde tanto si la integridad de la investigación se pone en riesgo como si el monitoreo no tiene importancia política y, por lo tanto, es un "mero ejercicio académico". Tal compromiso previo no depreciaría el rol de la política. Los valores y estándares se establecerían a través de un proceso esencialmente político. Además, las posibles respuestas a los resultados de ejercicios de monitoreo son, en su esencia, una cuestión de juicio político. No obstante, una vez que los ejercicios de monitoreo son avalados, constituyen constreñimientos sobre los políticos. Allí yace el mayor obstáculo potencial a la viabilidad política de propuestas para monitorear la democracia.

Algo bastante diferente es requerido por parte de los investigadores. Generar información rigurosa y creíble sobre la política requiere la adhesión a estrictos criterios metodológicos y, por lo 
tanto, estar abierto a descubrir cosas que van en contra de lo que uno piensa. Pero si investigadores van a hacer su parte en el monitoreo de la democracia, deben combinar la tendencia a buscar conocimientos nuevos sin considerar consecuencias con una evaluación de las consecuencias de sus investigaciones. Esto es, los investigadores deben combinar aquello que Max Weber llama una "ética de la convicción" con una "ética de la responsabilidad" (Gerth y Mills, 1946: 77-156), y pensar como cientistas sociales y también como ciudadanos. Alli yace el mayor obstáculo para aquellos investigadores que correctamente se preocupan por el impacto que el involucramiento en política pueda tener sobre los estándares científicos.

La institucionalización de una asociación entre políticos e investigadores es una cuestión complicada. De hecho, en lugar de trabajar en forma cercana, escuchando y aprendiendo el uno del otro, políticos e investigadores trabajan a menudo con propósitos contrapuestos y con mucha frecuencia simplemente se ignoran. Por lo tanto, el establecimiento de normas que aseguren la autonomía del componente de investigación de los ejercicios de monitoreo y que también definan el uso previsto de la información generada a través de la investigación es uno de los desafíos centrales que aún debe ser abordado.

\section{3. ¿Qué? Integrando esfuerzos de monitoreo}

Un tercer desafío consiste en la integración de los esfuerzos de monitoreo. La razón de este objetivo es que muchas iniciativas de monitoreo se enfocan sobre aspectos delimitados de la democracia y, por lo tanto, se exponen a la crítica que llaman la atención a ciertas cuestiones e implícitamente excluyen otras de manera sesgada. Como es bien sabido, aunque ciertos países que no son democráticos han hecho mucho por garantizar los derechos sociales de sus ciudadanos, otros países con tradiciones liberales fuertes han puesto más énfasis en derechos políticos que sociales. Pues la manera en que un país es evaluado puede depender en gran medida de la información específica que se destaca, una posibilidad que pone en duda la empresa entera de monitoreo.

La solución a este problema es la adopción de un enfoque holístico, lo cual implica un esfuerzo por integrar las iniciativas parciales de monitoreo de la democracia. Aun más, en la medida que la democracia puede ser considerada como sólo uno de múltiples valores políticos, como se sugiere previamente, incluso el monitoreo de la democracia concebido de manera amplia puede ser criticado por excluir preguntas sobre el estado de derecho, los derechos humanos, el desarrollo, la seguridad y otras cuestiones críticas. Por lo tanto, la magnitud de la tarea que tendría que abordarse es bastante abrumadora. Pero es una tarea factible.

Efectivamente, la adopción de un enfoque holístico contribuye a resolver uno de los problemas conceptuales más intratables que enfrentan los monitores, las aparentemente irresolubles disputas referidas a las definiciones tanto en la literatura académica como en las discusiones políticas. Cómo se definen los conceptos es una cuestión importante, entre otras cosas, porque tales definiciones proveen un punto de partida necesario para cualquier intento de medición. Pero las disputas sobre el concepto de la democracia se han vuelto debates terminológicos, en gran medida improductivos, acerca de cuál es la definición correcta de la democracia. Y la adopción de un enfoque holístico, aun si esto llevase sólo a la identificación de una simple lista, lógicamente organizada, de cuestiones relevantes, haría mucho por resolver varios desacuer- 
dos, como aquellos entre los que adoptan definiciones minimalistas y maximalistas. Por lo tanto, un desafío clave en los ejercicios de monitoreo es crear un mayor consenso acerca de la importancia de un enfoque holístico e integrar esfuerzos de monitoreo que se han encarado de manera fragmentada. De esta forma, se podrá pasar de la tarea de conceptualización a la de medición y así abrir la posibilidad de trascender debates conceptuales por medio de un análisis empírico y conclusiones sustantivas.

\section{4. ¿Cómo? Solucionando problemas de medición}

Finalmente, la resolución de problemas de medición constituye otro desafío. Mucho se ha aprendido, como se menciona antes, en referencia a cómo medir la democracia y otros conceptos relacionados. No obstante, algunos problemas aún no se han abordado satisfactoriamente. Primero, los umbrales utilizados en las escalas deben calibrarse mejor de forma de corresponderse con los estándares desarrollados y aceptados por la comunidad internacional. Segundo, las decisiones sobre codificación -esto es, de asignación de valores en cierta escala- deben realizarse de manera más consistente, pero también flexible, asegurando el doble objetivo de que los estándares sean aplicados de manera universal y también reconociendo la posibilidad de que problemas similares se manifiesten diferentemente en distintos países y a través del tiempo. Tercero, los procedimientos utilizados para agregar las mediciones de diferentes dimensiones de la democracia merecen una mayor discusión. Por ejemplo, aunque se ha vuelvo común hacer una distinción entre ser elegido democráticamente y gobernar democráticamente, se le otorga escasa atención al peso relativo de estas dimensiones en una evaluación global de la democracia. Cuarto, evaluaciones discordantes basadas en diferentes fuentes de datos deben ser reconciliadas 0 , por lo menos, explicadas. En resumen, es crucial reconocer que la medición de conceptos políticos como la democracia es una ciencia muy incierta y promover una mayor reflexión metodológica para así asegurar la producción de datos de alta calidad que puedan ser utilizados de manera responsable en el proceso político.

\section{CONCLUSIÓN}

El monitoreo de la democracia es tanto deseable como factible. Sin embargo, a medida que las propuestas de monitoreo de la democracia han logrado prominencia y lo que está en juego políticamente en estos ejercicios de monitoreo se ha vuelto más evidente, se han empezado a formular críticas a los intentos de monitoreo de la democracia. Algunos de los críticos buscan simplemente oponerse a la entera empresa de monitoreo y es probable que hay poco que pueda hacerse para satisfacer sus demandas, con excepción de renunciar a cualquier intento de monitoreo de la democracia. Sin embargo, otros críticos plantean argumentos legítimos, que merecen ser abordados seriamente. En consecuencia, para prevenir el crecimiento potencial de la oposición a ejercicios de monitoreo, es necesario enfrentar algunos desafíos fundamentales referidos al porqué, quiénes, qué y cómo del monitoreo.

Estos desafíos involucran temáticas complicadas: el reconocimiento de, y balance entre, múltiples valores políticos; el acercamiento de políticos e investigadores; la integración de esfuerzos de monitoreo, y la resolución de problemas de medición. Por lo tanto, una profundización del consenso que ha surgido en cuanto al monitoreo de la democracia a partir de la incorporación 
de respuestas adecuadas a estos desafíos requerirá una gran dosis de voluntad política y nuevas formas de pensar por parte de quienes promueven la democracia. Pero mucho depende de un esfuerzo colectivo sostenido para confrontar estos desafíos. Efectivamente, la manera en que estos se confronten con seguridad determinará si el ímpetu que se ha desarrollado en torno al monitoreo de la democracia se mantiene o comienza a debilitarse.

\section{REFERENCIAS}

Agencia de Estados Unidos para el Desarrollo Internacional (USAID). 1998. Handbook of Democracy and Governance Program Indicators. Washington, D.C.: Centro USAID para Ia Democracia y la Gobernabilidad, Serie de Publicaciones Técnicas PN-ACC-390.

Agencia de Estados Unidos para el Desarrollo Internacional (USAID). 2000. Conducting a DG Assesment: A Frame Work for Strategy Development. Washington, D.C.: Centro USAID para la Democracia y la Gobernabilidad, Serie de Publicaciones Técnicas PN-ACH-305.

Asamblea General de las Naciones Unidas. 1993. Declaración y Programa de Acción de Viena. A/CONF.157/23, 12 de julio de 1993.

Asamblea General de las Naciones Unidas. 2000. Declaración del Milenio. A/RES/55/2, 18 de septiembre de 2000.

Beetham, David, Sarah Bracking, lain Kearton y Stuart Weir (ed.). 2001. International IDEA Handbook on Democracy Assesment. La Haya: Kluge Academic Publishers.

Bjornlund, Eric C. 2004. Beyond Free and Fair: Monitoring Elections and Building Democracy. Baltimore: Johns Hopkins University Press.

Campbell, Kim y Sean C. Carroll. 2005. "Sustaining Democracy's Last Wave". Georgetown Journal of International Affairs 6 (2): 45-52.

Comisión Internacional sobre Intervención y Soberanía de los Estados. 2001. La responsabilidad de proteger. Ottawa: International Development Research Center.

Dahl, Robert A. 1999. La democracia. Una guía para los ciudadanos. Madrid: Taurus.

Elklit, Jørgen y Andrew Reynolds. 2005. “A Framework for The Systematic Study of Election Quality”. Democratization 12 (2): $147-162$

Garber, Larry. 1984. Guidelines for International Election Observing. Washington, D.C.: International Human Rights Law Group.

Gerth, Hans H. y C. Wright Mills (ed.). 1946. From Max Weber: Essays in Sociology. Nueva York: Oxford University Press. Krasner, Stephen. 2004. "Shared Sovereignity: New Institutions for Collapsed and Failing Status". International Security 29 (2): 85-120.

Landman, Todd y Julia Häuserman. 2003. Map-Making and Analysis of The Main International Initiatives on Developing Indicators on Democracy and Good Governance. Luxemburgo: Oficina de Estadística de la Comunidad Europea, EUROSTAT.

McFaul, Michael. 2004. “Democracy Promotion as a World Value”. The Washington Quarterly 28 (1): 147-163.

Munck, Gerardo L. y Jay Verkuilen. 2002. "Conceptualizando y midiendo la democracia: Una Evaluación de Índices Alternativos". Política y Gobierno 9 (2): 403-441.

Munck, Gerardo L. 2005. “Measuring Democratic Governance: Central Tasks and Basic Problems”. En Measuring Empowerment: Cross-Disciplinary Perspectivas, editado por Deepa Narayan. Washington, DC: Banco Mundial, 427-459.

Munck, Gerardo L. 2006. "Drawing Boundaries: How to Craft Intermediate Regime Categories". En Electoral Authoritarism: The Dynamics of Unfree Competition, editado por Andreas Schedler. Boulder, Col.: Lynne Rienner Publishers, 35-57.

Organización de Estados Americanos. 2001. Carta Democrática Interamericana. Washington, DC.: OEA.

Pastor, Robert A. 2003. "A Community of Democracies in The Americas: Instilling Substance into a Wondrous Phrase". Canadian Foreign Policy 10 (3): 15-29.

Piccone, Theodore J. 2005. "International Mechanisms for Protecting Democracy". En Protecting Democracy: International Responses, editado por Morton H. Halperin y Mirna Galic. Lanham, MD.: Lexington Books, 101-126.

Programa de las Naciones Unidas para el Desarrollo (PNUD). 2002. Informe sobre Desarrollo Humano 2002. Profundizar la democracia en un mundo fragmentado. Nueva York y Madrid: PNUD y Ediciones Mundi-Prensa.

Programa de las Naciones Unidas para el Desarrollo (PNUD). 2004. La democracia en América Latina. Hacia una democracia de ciudadanas y ciudadanos. Nueva York y Buenos Aires: PNUD y Aguilar, Altea, Taurus, Alfaguara.

Przeworski, Adam. 1997. "Una defensa de la concepción minimalista de la democracia”. Revista Mexicana de Sociología 59 (3): 3-36. 
Przeworski, Adam, Michael E. Álvarez, José Antonio Cheibub y Fernando Limongi. 2000. Democracy and Development: Political Institutions and Well-Being in The World, 1950-1990. Nueva York: Cambridge University Press.

Rich, Roland. 2001. "Bringing Democracy into International Law”. Journal of Democracy 12 (3): 20-34.

Secretario General de las Naciones Unidas. 2005. Un concepto más amplio de la libertad: Desarrollo, seguridad y derechos humanos para todos. Informe del Secretario General, A/59/2005.

Sen, Amartya. 2001. "La democracia como valor universal”. Istor 1 (4): 10-28.

Scheuch, Erwin K. 2003. "Historia y perspectivas de los servicios de datos utilizados en ciencias sociales". Revista Internacional de Ciencias Sociales 55 (177): 18-35.

Diario Oficial de la Unión Europea. 10 de noviembre, 1997. C 340. "Tratado de Amsterdam por el que se modifican el Tratado de la Unión Europea, los tratados constitutivos de las comunidades europeas y determinados actos conexos".

Gerardo L. Munck es profesor en la Escuela de Relaciones Internacionales de la Universidad del Sur de California (USC), y se dedica a la investigación sobre regímenes políticos y democracia, metodología, y América Latina. Actualmente, se encuentra editando Regímenes y Democracia en América Latina, y acaba de completar (con Richard Snyder) un libro que consiste en entrevistas con renombrados académicos, Pasión, oficio y método en la política comparada. Fue el Consultor Técnico en Jefe, responsable por la preparación del compendio estadístico del informe del Programa de las Naciones Unidas para el Desarrollo (PNUD), La Democracia en América Latina.

(E-mail: munck@usc.edu) 